\title{
Nonlinear Differential Equations with Exact Solutions Expressed via the Weierstrass Function
}

\author{
Nikolai A. Kudryashov \\ Department of Applied Mathematics Moscow Engineering and Physics Institute (State university), \\ 31 Kashirskoe Shosse, 115409 Moscow, Russian Federation \\ Reprint requests to Prof. N. A. K.; E-mail:kudr@dampe.mephi.ru \\ Z. Naturforsch. 59a, 443 - 454 (2004); received March 22, 2004 \\ A new problem is studied, that is to find nonlinear differential equations with special solutions \\ expressed via the Weierstrass function. A method is discussed to construct nonlinear ordinary dif- \\ ferential equations with exact solutions. The main step of our method is the assumption that nonlin- \\ ear differential equations have exact solutions which are general solution of the simplest integrable \\ equation. We use the Weierstrass elliptic equation as building block to find a number of nonlinear \\ differential equations with exact solutions. Nonlinear differential equations of the second, third and \\ fourth order with special solutionsexpressed via the Weierstrass function are given. - PACS: $02.30 . \mathrm{Hq}$ \\ (Ordinary differential equations)
}

Key words: Nonlinear Differential Equation; Exact Solution; Weierstrass Function; Nonlinear Evolution Equation.

\section{Introduction}

Many papers with methods of finding exact solutions of nonlinear differential equations [1-9] were published in last time.

Nonlinear differential equations can be divided into three types: exactly solvable, partially solvable, and unsolvable.

Usually investigators solve two problems. The first problem is to find nonlinear differential equations that have solutions, and the second problem is to find such solutions.

This paper is devoted to the solution of a new problem, that is to find nonlinear differential equations with special solutions. It is important to note that these equations have exact solutions, but they are not all integrable equations. Using our approach, we extend the class of studied differential equations.

Consider the nonlinear evolution equation

$$
E_{1}[u] \equiv E_{1}\left(u, u_{t}, u_{x}, \ldots, x, t\right)=0 .
$$

Assume we need exact solutions of this equation. Usually we look for exact solutions of nonlinear evolution equations taking the travelling wave into account and search exact solution of (1.1) in the form

$$
u(x, t)=y(z), \quad z=x-C_{0} t .
$$

As a result (1.1) reduces to the nonlinear ordinary differential equation (ODE)

$$
E_{2}[y] \equiv E_{2}\left(y, y_{z}, \ldots, z\right)=0 .
$$

To obtain exact solutions of (1.3) one can apply different approaches [10-21]. However, most of the methods used to find exact solutions take the singular analysis for solutions of nonlinear differential equations into account.

Using the singular analysis, first of all one can consider the leading members of (1.3). After that one can find the singularity for the solution of (1.3). Further the truncated expansion is used to have the transformation to search exact solutions of nonlinear ODEs. At this point one can use some trial functions (hyperbolic, elliptic and so on) to look for exact solutions of nonlinear ODEs.

However, one can note that hyperbolic and elliptic functions are general solutions of nonlinear exactly solvable equations. We have as a rule that partially solvable nonlinear differential equations have exact solutions that are general solutions of solvable equations of lesser order.

This paper is an extension of [22], where we started to find nonlinear ordinary differential equations of polynomial form that have special solutions expressed via general solutions of the Riccati equation. 
Let us explain the idea of this work. As is well known, there is the great problem to find integrable nonlinear differential equations. However sometimes we can content ourselves with some special solutions, because many differential equations are nonintegrable although they are intensively used in physics and look as simple equations. It is important to find special solutions of these equations that are called exact solutions. In this paper we want to find nonlinear differential equations that are not all integrable but have special solutions in the form of the Weierstrass function.

The first aim of this work is to present the method to find nonlinear differential equations with exact solutions in the form of the Weierstrass function. The second aim is to give nonlinear ordinary differential equations that have exact solutions expressed via the general solution of the Weierstrass elliptic equation.

The outline of this paper is as follows: In Sect. 2 we present the method to find nonlinear ODEs with special solutions, expressed via the Weierstrass function. Nonlinear ODEs with exact solutions of the first, second, third and fourth degree singularities are in Sect. 3, 4, 5 and 6. An example of a nonlinear ODE with exact solution of the fifth degree singularity, that is popular in the description of the model chaos, is considered in Sect. 7.

\section{Applied Method}

Let us discuss the method to find nonlinear differential equations with exact solutions expressed via the Weierstrass function: Most nonlinear ODEs have exact solutions that are general solutions of differential equations of lesser order. Most nonlinear differential equations are determined via general solutions of the Riccati equation. This is so because most approaches to search exact solutions of nonlinear ODEs are based on general solutions of the Riccati equation. The application of the tanh method confirms this idea [18-21]. We gave these nonlinear differential equations in [22].

However many nonlinear ODEs have exact solutions that are general solutions of the Weierstrass elliptic functions $[1,12,14,15,23-25]$ and the Jacobi elliptic functions $[3,8,26,27]$. In this paper we are going to find nonlinear ODEs with exact solutions expressed via the Weierstrass function.

The Weierstrass elliptic equation can be presented in the form

$$
P_{1}[R]=R_{z}^{2}-4 R^{3}+g_{2} R+g_{3}=0 .
$$

We have the following simple theorem.

Theorem 2.1. Let $R(z)$ be a solution of Eq. (2.1). Than the equations

$$
\begin{aligned}
& R_{z z}=6 R^{2}-\frac{1}{2} g_{2}, \\
& R_{z z z}=12 R R_{z} \\
& R_{z z z z}=120 R^{3}-18 g_{2} R-12 g_{3}, \\
& R_{z z z z z}=360 R^{2} R_{z}-18 g_{2} R_{z},
\end{aligned}
$$

have special solutions expressed via the general solution of (2.1).

Proof. Theorem 2.1 is proved by differentiation of (2.1) with respect to $z$ and substitution $R_{z}^{2}$ from (2.1) and so on into expressions obtained.

The general solution of (2.1) is the Weierstrass function

$$
R(z)=\wp\left(z, g_{2}, g_{3}\right)
$$

where $g_{2}$ and $g_{3}$ are arbitrary constants that are called invariants.

It is known that the Weierstrass elliptic function can be found via the Jacobi elliptic functions. For example let us demonstrate that the solution $R(z)$ of (2.1) is expressed via the Jacobi elliptic function $\operatorname{sn}(x, k)$.

Taking the transformation

$$
R\left(z, g_{2}, g_{3}\right)=k^{2} X(z)^{2}-\frac{1}{3}\left(1+k^{2}\right)
$$

with

$$
\begin{aligned}
& g_{2}=\frac{3}{4}\left(k^{4}-k^{2}+1\right), \\
& g_{3}=\frac{4}{9}\left(\frac{2}{3} k^{6}-k^{4}-k^{2}+\frac{2}{3}\right)
\end{aligned}
$$

into account, we have

$$
X_{z}^{2}=\left(1-X^{2}\right)\left(1-k^{2} X^{2}\right)
$$

The solution of (2.10) is the Jacobi elliptic function

$$
X(z)=\operatorname{sn}(x, k) .
$$

We want to find nonlinear ODEs which have special solutions that are determined via the general solution of the Weierstrass elliptic equation. 
The algorithm of our method can be presented by four steps. At the first step we choose the singularity of the special solution and give the form of this solution. For example we can see that the singularity of the first degree with the Weierstrass function takes the form

$$
y(z)=\frac{R_{z}}{R} .
$$

However the special solution with singularity of the second degree has the form

$$
y(z)=R(z)
$$

At this step we have to take a more general form of the exact solution into account.

At the second step we set the order of the nonlinear ODE which we want to search. For example, using (2.8) we can take the ODE of the fourth order, where the leading members take the form

$$
a_{0} y_{z z z z}+a_{1} y y_{z z}+a_{2} y_{z}^{2}+a_{3} y^{3}=0
$$

The third step lies in the fact that we write the general form of the nonlinear differential equation, taking the singularity of the solution and the given order for the nonlinear differential equation into account.

The fourth step contains calculations taking theorem 2.1 into account. As a result we find limitations for the parameters so that the nonlinear differential equation has exact solutions. At this step we can have nonlinear ODE with exact solutions.

As this takes place, we have nonlinear ODEs with special solutions in the form of periodic functions. These solutions are important because many nonlinear evolution equations present periodical waves expressed via the Weierstrass elliptic function.

\section{Nonlinear ODEs with Exact Solutions of the First Degree Singularity}

Let us demonstrate our approach to find nonlinear ODEs with exact solutions of a first degree singularity. These solutions can be presented in the form

$$
y(z)=A_{0}+A_{1} \frac{R_{z}}{R} .
$$

Here $R(z)$ is a solution of the Weierstrass elliptic equation, while $A_{0}$ and $A_{1}$ are unknown constants. First of all we are going to find nonlinear second order ODEs.
Second order ODEs. General form of the second order ODEs with solution (3.1) can be presented as

$$
y_{z z}+a_{1} y y_{z}+a_{2} y^{3}+b_{0} y_{z}+b_{1} y^{2}-C_{0} y+C_{1}=0 .
$$

Equation (3.2) was written taking a singularity of the solution (3.1) into account and the given order of the nonlinear ODE for which we want to have exact solutions.

Let us assume $A_{1}=1$. Substituting $y(z)$ from (3.1) into (3.2) and using (2.1) and (2.2) we have an equation in the form

$$
\begin{aligned}
& \left(2 b_{0}+12 a_{2} A_{0}+4 b_{1}+2 a_{1} A_{0}\right) R^{4} \\
& +\left(4 a_{2} R_{z}+2 R_{z}+a_{2} A_{0}^{3}-C_{0} A_{0}+2 a_{1} R_{z}+C_{1}+b_{1} A_{0}^{2}\right) R^{3} \\
& +\left(2 b_{1} A_{0} R_{z}-3 a_{2} A_{0} g_{2}-b_{1} g_{2}+3 a_{2} A_{0}^{2} R_{z}-C_{0} R_{z}\right) R^{2} \\
& +\left(b_{0} g_{3}+a_{1} A_{0} g_{3}-a_{2} R_{z} g_{2}-3 a_{2} A_{0} g_{3}-b_{1} g_{3}\right) R \\
& -a_{2} R_{z} g_{3}-2 R_{z} g_{3}+a_{1} R_{z} g_{3}=0 .
\end{aligned}
$$

From (3.3) we get relations for the constants:

$$
\begin{gathered}
a_{1}=-1-2 a_{2}, \quad C_{0}=3 a_{2} A_{0}^{2}+2 b_{1} A_{0}, \quad g_{2}=0, \\
a_{2}=-1, \quad A_{0}=\frac{1}{5} b_{0}+\frac{2}{5} b_{1}, \\
C_{1}=-\frac{7}{125} b_{0}^{2} b_{1}-\frac{4}{125} b_{0} b_{1}^{2}+\frac{4}{125} b_{1}^{3}-\frac{2}{125} b_{0}^{3}, \\
b_{1}=-3 b_{0} .
\end{gathered}
$$

Taking these constants into account, we have

$$
y_{z z}+y y_{z}-y^{3}+b_{0} y_{z}-3 b_{0} y^{2}-3 b_{0}^{2} y-b_{0}^{3}=0 .
$$

The solution of (3.7) is expressed by the formula

$$
y(z)=-b_{0}+\frac{R_{z}}{R}
$$

Periodic solutions of (3.8) of equation (3.7) are demonstrated in Fig. 1 for $b_{0}=-12.0, g_{2}=0.5625$ and $g_{3}=0.015$.

For $b_{0}=0$, from (3.7) we have an equation that was found by Painleve [28].

Third order ODEs. The general case of third order ODEs with exact solutions (3.1) takes the form

$$
\begin{gathered}
a_{0} y_{z z z}+a_{1} y y_{z z}+a_{2} y_{z}^{2}+a_{3} y^{4}+b_{0} y_{z z}+b_{1} y y_{z} \\
+b_{2} y^{3}++d_{0} y_{z}+d_{1} y^{2}-C_{0} y+C_{1}=0 .
\end{gathered}
$$




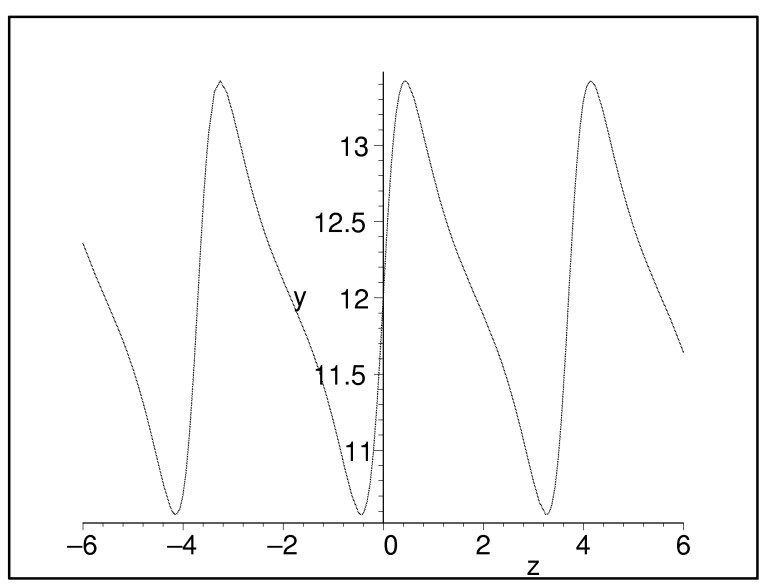

Fig. 1. Periodic solution (3.8) of Eq. (3.7) at $b_{0}=-12.0$, $g_{2}=0.5625$ and $g_{3}=0.015$.

Assuming $A_{1}=1$ without loss of the generality, and substituting (3.1) into (3.9), we get the following coefficients:

$$
\begin{gathered}
b_{2}=-\frac{1}{2} a_{1} A_{0}-\frac{1}{2} b_{1}-\frac{1}{2} b_{0}-4 a_{3} A_{0}, \\
C_{0}=-\frac{3}{2} b_{1} A_{0}^{2}+2 d_{1} A_{0}-8 a_{3} A_{0}^{3} \\
\quad-\frac{3}{2} A_{0}^{3} a_{1}-\frac{3}{2} A_{0}^{2} b_{0}, \\
b_{1}=0, \quad b_{0}=-a_{1} A_{0}, \\
a_{3}=-\frac{1}{4} a_{2}-\frac{3}{4} a_{0}-\frac{1}{2} a_{1}, \\
d_{1}=-\frac{1}{2} d_{0}-\frac{3}{2} A_{0}^{2} a_{2}-\frac{9}{2} A_{0}^{2} a_{0}-3 A_{0}^{2} a_{1}, \\
C_{1}=-4 g_{2} a_{2}-6 a_{0} g_{2}-2 a_{1} g_{2}-\frac{1}{4} A_{0}^{4} a_{2} \\
-\frac{3}{4} A_{0}^{4} a_{0}-\frac{1}{2} A_{0}^{4} a_{1}-\frac{1}{2} A_{0}^{2} d_{0}, \\
a_{2}=-\frac{1}{6} \frac{6 a_{1} g_{3}+18 a_{0} g_{3}+g_{2} d_{0}}{g_{3}}, \\
d_{0}=\frac{a_{0} g_{2}^{2}}{g_{3}}, \\
a_{1}=\frac{a_{0}\left(g_{2}^{3}+108 g_{3}^{2}\right)}{24 g_{3}^{2}} .
\end{gathered}
$$

We also get two relations for constants $g_{3}$ :

$$
g_{3}= \pm \frac{1}{6} g_{2}^{3 / 2}
$$

As a result, we have the following equations:

$$
\begin{aligned}
y_{z z z} & +\left(6 y-6 A_{0}\right) y_{z z}-3 y_{z}^{2}-3 y^{4}+12 A_{0} y^{3} \\
& +\left(-18 A_{0}^{2} \mp 3 \sqrt{g_{2}}\right) y^{2}+\left( \pm 6 A_{0} \sqrt{g_{2}}+12 A_{0}^{3}\right) y \\
& \mp 3 A_{0}^{2} \sqrt{g_{2}}-3 A_{0}^{4} \pm 6 \sqrt{g_{2}} y_{z}+6 g_{2}=0
\end{aligned}
$$

with exact solutions

$$
y(z)=A_{0}+\frac{R_{z}}{R}
$$

Fourth order ODEs. Now let us find nonlinear fourth order ODEs with exact solutions of the first degree singularity. We have the following nonlinear fourth order ODEs of the general form

$$
\begin{aligned}
a_{0} y_{z z z z} & +a_{1} y y_{z z z}+a_{2} y^{2} y_{z z}+a_{3} y^{3} y_{z}+a_{4} y^{5} \\
& +a_{5} y y_{z}^{2}+a_{6} y_{z} y_{z z}+b_{0} y_{z z z}+b_{1} y y_{z z} \\
& +b_{2} y_{z}^{2}+b_{3} y^{4}+d_{0} y_{z}+d_{1} y y_{z}+d_{2} y^{3} \\
& +h_{0} y_{z}+h_{1} y^{2}-C_{0} y+C_{1}=0 .
\end{aligned}
$$

Assuming $g_{2}=m^{2}$ and substituting (3.1) at $A_{0}=0$ and $A_{1}=1$ into (3.21) we get coefficients in the form

$$
\begin{aligned}
a_{6}= & -6 a_{0}-3 a_{1}-4 a_{4}-2 a_{2}-2 a_{3}-a_{5}, \\
d_{2}= & -\frac{1}{2} d_{1}-\frac{1}{2} d_{0}, \\
C_{0}= & -4 a_{2} m^{2}-8 a_{4} m^{2}+2 a_{5} m^{2}, \\
a_{5}= & a_{2}-a_{3}-3 a_{1}-\frac{1}{6} \frac{m^{2} d_{1}}{g_{3}}, \\
a_{4}= & -\frac{3}{2} a_{0}+\frac{3}{4} a_{1}-\frac{3}{4} a_{2}+\frac{1}{4} a_{3} \\
& +\frac{3}{4} \frac{d_{0} g_{3}}{m^{4}}-\frac{3}{4} \frac{d_{1} g_{3}}{m^{4}}+\frac{1}{24} \frac{d_{1} m^{2}}{g_{3}}, \\
a_{3}= & -6 \frac{d_{0} g_{3}}{m^{4}}+\frac{1}{12} \frac{d_{1} m^{2}}{g_{3}}+3 a_{1} \\
& -15 a_{0}+6 \frac{d_{1} g_{3}}{m^{4}}-2 a_{2}, \\
a_{2}= & -5 a_{0}+2 a_{1}+\frac{1}{36} \frac{d_{1} m^{2}}{g_{3}} \\
& \quad-3 \frac{d_{0} g_{3}}{m^{4}}+3 \frac{d_{1} g_{3}}{m^{4}}, \\
b_{3}= & -\frac{3}{4} b_{0}-\frac{1}{4} b_{2}-\frac{1}{2} b_{1}, \quad h_{1}=-\frac{1}{2} h_{0},
\end{aligned}
$$




$$
\begin{aligned}
& C_{1}=-6 b_{0} m^{2}-4 b_{2} m^{2}, \\
& b_{2}=b_{1}-3 b_{0}-\frac{1}{6} \frac{h_{0} m^{2}}{g_{3}}, \\
& h_{0}=\frac{b_{0} m^{4}}{g_{3}} \\
& b_{0}=\frac{1}{16} \frac{h_{0} g_{3}}{m^{4}}+\frac{1}{36} \frac{h_{0} m^{2}}{g_{3}}, \\
& g_{3}^{(1,2)}= \pm \frac{1}{6} m^{3} .
\end{aligned}
$$

As a result we have at $g_{3}=\frac{1}{6} m^{3}$ an equation of the form

$$
\begin{aligned}
a_{0} y_{z z z z} & +\frac{1}{6}\left(6 a_{1} y+b_{1}\right) y_{z z z} \\
& +\left(10 a_{0}-a_{1}+\frac{1}{2} \frac{d_{0}}{m}-\frac{1}{6} \frac{d_{1}}{m}\right) y_{z} y_{z z} \\
& +\left(d_{0}+b_{1} y\right) y_{z z} \\
& +\left(2 a_{1}-\frac{2}{3} \frac{d_{1}}{m}+\frac{1}{2} \frac{d_{0}}{m}-5 a_{0}\right) y^{2} y_{z z} \\
& +\left(\frac{1}{2} \frac{d_{1}}{m}+\frac{1}{2} \frac{d_{0}}{m}+b_{2}\right) y y_{z}^{2} \\
& +\left(a_{0}-\frac{1}{2} \frac{d_{0}}{m}-a_{1}+\frac{1}{3} \frac{d_{1}}{m}\right) y^{5} \\
& -\left(\frac{1}{4} b_{2}+\frac{5}{8} b_{1}\right) y^{4}-\left(\frac{1}{2} d_{1}+\frac{1}{2} d_{0}\right) y^{3} \\
& +\frac{1}{2} b_{1} m y^{2} \\
& +\left(d_{1} y-5 y^{3} a_{0}-y^{3} a_{1}-\frac{1}{6} \frac{y^{3} d_{1}}{m}-b_{1} m\right) y_{z} \\
& -\left(m d_{1}+12 m^{2} a_{0}-3 m d_{0}\right) y \\
& -4 b_{2} m^{2}-m^{2} b_{1}=0
\end{aligned}
$$

with an exact solution of the form

$$
y(z)=\frac{R_{z}}{R},
$$

when $R(z)$ is the solution of an equation of the form

$$
R_{z}^{2}-4 R^{3}+m^{2} R+\frac{1}{6} m^{3}=0 .
$$

The solution of (3.35) is expressed via the Weierstrass function and has a first degree singularity. At the present we do not know any application of (3.35).

\section{Nonlinear ODEs with Exact Solutions of the Second Degree Singularity}

Let us find nonlinear ODEs with exact solutions of the second degree singularity, expressed via the Weierstrass function

$$
y(z)=A_{0}+A_{1} \frac{R_{z}}{R}+A_{2} R .
$$

We do not consider the second order nonlinear ODEs with exact solutions (4.1), because this equation is exactly solvable. We start to find the third order nonlinear ODEs with exact solutions of the second degree singularity, expressed via the Weierstrass function.

Third order ODEs. Let us write the general form of the nonlinear third ODEs with solution of the second order singularity. It takes form

$$
a_{0} y_{z z z}+a_{1} y y_{z}+b_{0} y_{z z}+b_{1} y^{2}+d_{0} y_{z}-C_{0} y+C_{1}=0 .
$$

Substituting (4.1) into (4.2) we obtain the following parameters:

$$
\begin{aligned}
& a_{1}=-12 a_{0}, \quad d_{0}=12 a_{0} A_{0}, \quad b_{1}=\frac{C_{0}}{2 A_{0}}, \\
& A_{1}=0, \quad A_{0}=-\frac{C_{0}}{12 b_{0}}, \\
& g_{2}=\frac{C_{0}^{2}+24 C_{1} b_{0}}{12 b_{0}^{2}} .
\end{aligned}
$$

As a result we have an equation of the form

$$
\begin{gathered}
a_{0} y_{z z z}-12 a_{0} y y_{z}+b_{0} y_{z z}-\frac{a_{0} C_{0}}{b_{0}} y_{z} \\
-6 b_{0} y^{2}-C_{0} y+C_{1}=0
\end{gathered}
$$

with exact solutions

$$
y(z)=-\frac{C_{0}}{12 b_{0}}+R(z) .
$$

A periodic solution of (4.7) that is determined by (4.6) at $C_{0}=-12, b_{0}=1, g_{2}=0.7485$ and $g_{3}=-0.2954$ is given in Figure 2.

Equation (4.6) can be found from the nonlinear evolution equation

$$
\begin{gathered}
u_{t}+\lambda_{1} u u_{x}+\lambda_{2}\left(u u_{x}\right)_{x}+\lambda_{3} u_{x x} \\
+\lambda_{4} u_{x x x}+\lambda_{5} u_{x x x x}=0 .
\end{gathered}
$$




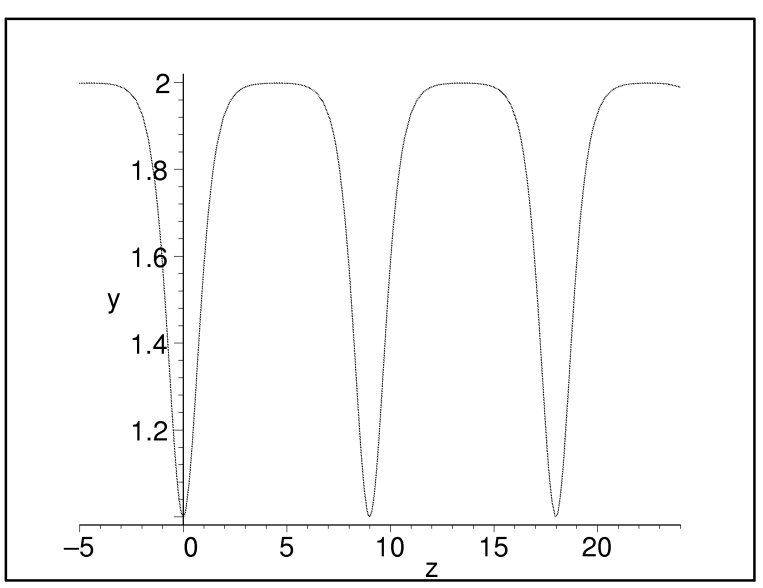

Fig. 2. Periodic solution (4.7) of Eq. (4.6) with $C_{0}=-12$, $b_{0}=1, g_{2}=0.7485$ and $g_{3}=-0.2954$.

The nonlinear evolution equation (4.8) was used at the description of nonlinear wave processes and was studied in works [29-32]. Exact solutions of this equation were obtained in $[33,34]$. They are also rediscovered later.

Fourth order ODEs. Now let us find the nonlinear fourth order ODEs with exact solutions of the second degree singularity expressed via the Weierstrass function. The general case of this nonlinear ODE can be presented in the form

$$
\begin{gathered}
a_{0} y_{z z z z}+a_{1} y y_{z z}+a_{2} y_{z}^{2}+a_{3} y^{3}+b_{0} y_{z z z}+b_{1} y y_{z} \\
+d_{0} y_{z z}+d_{1} y^{2}+h_{0} y_{z}-g_{2} C_{0} y+C_{1}=0 .
\end{gathered}
$$

We assume that exact solutions of (4.9) can be found by (4.1).

Without loss of generality let us assume in (4.1) $A_{0}=0$ and $A_{2}=1$. We obtain the following parameters for (4.9)

$$
\begin{aligned}
& b_{1}=-12 b_{0}, \quad h_{0}=0, \quad A_{1}=0, \\
& a_{3}=-120 a_{0}-6 a_{1}-4 a_{2}, \quad d_{1}=-6 d_{0}, \\
& a_{2}=-18 a_{0}-\frac{1}{2} a_{1}-C_{0}, \\
& C_{1}=\frac{1}{2} d_{0} g_{2}-\frac{1}{2} g_{3} a_{1}-6 a_{0} g_{3}-g_{3} C_{0} .
\end{aligned}
$$

We get nonlinear ODEs in the form

$$
\begin{aligned}
a_{0} y_{z z z z} & +b_{0} y_{z z z}+\left(a_{1} y+d_{0}\right) y_{z z} \\
& -\left(18 a_{0}+\frac{1}{2} a_{1}+C_{0}\right) y_{z}^{2}-12 b_{0} y y_{z}
\end{aligned}
$$

$$
\begin{aligned}
& +\left(4 C_{0}-48 a_{0}-4 a_{1}\right) y^{3}-6 d_{0} y^{2} \\
& -g_{2} C_{0} y+\frac{1}{2} d_{0} g_{2}-\frac{1}{2} g_{3} a_{1}-6 a_{0} g_{3} \\
& -g_{3} C_{0}=0 .
\end{aligned}
$$

Exact solutions of (4.14) are found by the formula

$$
y(z)=R(z) .
$$

Assuming $a_{0}=1, b_{0}=0, d_{0}=0$ in (4.14) we have

$$
\begin{aligned}
y_{z z z z} & +a_{1} y y_{z z}-\left(18+\frac{1}{2} a_{1}+C_{0}\right) y_{z}^{2} \\
& +4\left(C_{0}-12-a_{1}\right) y^{3}-g_{2} C_{0} y-6 g_{3} \\
& -\frac{1}{2} g_{3} a_{1}-g_{3} C_{0}=0 .
\end{aligned}
$$

One can note that (4.16) has two parameters in the leading members. However let us show that this equation contains many important nonlinear integrable and nonintegrable differential equations.

Assuming $a_{1}=-30, C_{0}=-3$ and $y(z) \rightarrow-y(z)$ in (4.16) we have an equation in the form

$$
y_{z z z z}+30 y y_{z z}+60 y^{3}+3 g_{2} y-12 g_{3}=0 .
$$

Equation (4.17) is exactly solvable and can be obtained by the travelling wave (1.2) from the Caudrey-DoddGibbon equation $[35,36,38]$

$$
u_{t}+\frac{\partial}{\partial x}\left(u_{x x x x}+30 u u_{x x}+60 u^{3}\right)=0 .
$$

Equation (4.18) is integrable by the inverse scattering transform. However we can see that this equation is found among our class of nonlinear differential equations.

Assuming $a_{1}=-20, C_{0}=2$ and $y(z) \rightarrow-y(z)$ in (4.16), we have

$$
\begin{aligned}
y_{z z z z} & +20 y y_{z z}+10 y_{z}^{2}+40 y^{3} \\
& -2 g_{2} y-2 g_{3}=0 .
\end{aligned}
$$

Using the travelling wave, we can see that (4.19) is obtained from the Korteveg - de Vries equation of the fifth order $[36,38]$

$$
u_{t}+\frac{\partial}{\partial x}\left(u_{x x x x}+20 u u_{x x}+10 u_{x}^{2}+40 u^{3}\right)=0 .
$$

We have shown that special solutions of the fifth order Korteveg - de Vries equation can be found by the formula (4.15). 
Assuming $a_{1}=-15, C_{0}=\frac{3}{4}$ and $y(z) \rightarrow-\frac{2}{3} y(z)$ in (4.16) we have an equation with an exact solution (4.15) in the form

$$
\begin{gathered}
y_{z z z z}+10 y y_{z z}+\frac{15}{2} y_{z}^{2}+\frac{20}{3} y^{3}-\frac{3}{4} g_{2} y \\
-\frac{9}{8} g_{3}=0 .
\end{gathered}
$$

Equation (4.21) is an exactly solvable equation too. Using the travelling wave, this one can be obtained from the Kaup - Kupershmidt equation [36-38]

$u_{t}+\frac{\partial}{\partial x}\left(u_{x x x x}+10 u u_{x x}+\frac{15}{2} u_{x}^{2}+\frac{20}{3} u^{3}\right)=0$.

Assuming $a_{1}=-15, C_{0}=\frac{3}{4}$ and $y(z) \rightarrow-y(z)$ in (4.16) we have

$$
\begin{gathered}
y_{z z z z}-15 y y_{z z}+\frac{45}{4} y_{z}^{2}+15 y^{3}-\frac{3}{4} g_{2} y \\
-\frac{3}{4} g_{3}=0 .
\end{gathered}
$$

Equation (4.23) is also exactly solvable [39]. This one has an exact solution (4.15) as well. This equation can be found from the Schwarz-Kaup-Kuperschmidt equation of the fifth order, that is the singular manifold equation for the Kaup-Kuperschmidt equation. The Cauchy problems for these equations can be solved by inverse scattering transform.

Assuming $a_{1}=-12, C_{0}=0$ and $y(z) \rightarrow-y(z)$ in (4.16) we have

$$
y_{z z z}+12 y y_{z z}+12 y_{z}^{2}=0 .
$$

Equation (4.24) is exactly solvable again [39]. The general solution of this equation is expressed via the first Painleve transcendent. However this equation has a special solution expressed by (4.15) as well.

We have interesting result that a number of exactly solvable equations exist in our class of differential equations. Many more nonlinear ODEs have similar special solutions expressed by (4.15) via the Weierstrass function. In future work we are going to look for exactly solvable nonlinear ODEs of higher order.

Assuming $a_{1}=0$ and $C_{0}=-18 a_{0}$ in (4.14), we have

$$
\begin{aligned}
a_{0} y_{z z z z} & +b_{0} y_{z z z}+d_{0} y_{z z}-12 b_{0} y y_{z}-120 y^{3} a_{0} \\
& -6 d_{0} y^{2}+18 g_{2} a_{0} y+C_{1}=0 .
\end{aligned}
$$

At $b_{0}=0$ we have nonlinear ODEs

$$
\begin{gathered}
a_{0} y_{z z z z}+d_{0} y_{z z}-120 a_{0} y^{3}-6 d_{0} y^{2} \\
+48 g_{2} a_{0} y+C_{1}=0 .
\end{gathered}
$$

Equation (4.26) corresponds to the nonlinear evolution equation that is used for the description of the nonlinear dispersive waves [40-43]

$$
u_{t}+\alpha u u_{x}+\beta u^{2} u_{x}+\gamma u_{x x x}+\delta u_{x x x x x}=0 .
$$

We have equation (4.26) if we look for an exact solution of (4.27) in the form of a travelling wave (1.2).

In the case $d_{0}=0$ of (4.25) we obtain nonlinear ODEs in the form

$$
a_{0} y_{z z z z}-120 a_{0} y^{3}+48 a_{0} g_{2} y+C_{1}=0 .
$$

Equation (4.28) is used for the description of nonlinear dispersive waves as well. The corresponding nonlinear evolution equation can be met at the description of nonlinear waves and takes the form $[44,45]$

$$
u_{t}+\beta u^{2} u_{x}+\delta u_{x x x x x}=0 .
$$

Exact solutions of (4.25), (4.26) and (4.28) are found by the formula (4.15).

\section{Nonlinear ODEs with the Third Degree Singularity Solution}

In this section we are going to find nonlinear ODEs that have exact solutions of the third degree singularity and are expressed via the Weierstrass function. These solutions can be presented by the formula

$$
y(z)=A_{0}+A_{1} \frac{R_{z}}{R}+A_{2} R+A_{3} R_{z} .
$$

We can not suggest any nonlinear ODEs of the second order with exact solutions (5.1) because we can not have the polynomial class of this equation. Let us start to consider the third nonlinear ODE.

Third order ODE. We have the general case of the nonlinear third order ODE in the form

$$
a_{0} y_{z z z}+a_{1} y^{2}+b_{0} y_{z z}+d_{0} y_{z}-C_{0} y+C_{1}=0 .
$$

Assuming $A_{3}=1$ and $g_{2}=\frac{n^{2}}{12}$, we have the following relations for the parameters:

$$
d_{0}=-2 a_{1} A_{1}+6 \frac{b_{0} A_{1}}{A_{2}}+\frac{C_{0}}{A_{2}}-2 \frac{a_{1} A_{0}}{A_{2}}
$$




$$
\begin{aligned}
& A_{1}=0, \quad b_{0}=-a_{0} A_{2}-\frac{1}{6} a_{1} A_{2}, \\
& a_{1}=-30 a_{0}, \quad C_{0}=-a_{0}\left(60 A_{0}-A_{2}^{3}\right) .
\end{aligned}
$$

We obtain four values for the constant $A_{2}$ :

$$
A_{2}^{(1,2)}= \pm n, \quad A_{2}^{(3,4)}= \pm i n
$$

and four values for the constant $C_{1}$ :

$$
\begin{aligned}
& C_{1}^{(1,2)}=-\frac{a_{0}}{24}\left(432 g_{3}\right.+720 A_{0}^{2} \\
&\left.\mp 24 A_{0} n^{3}-5 n^{6}\right), \\
& C_{1}^{(3,4)}=i \frac{a_{0}}{24}\left(432 i g_{3}+\right. 720 i A_{0}^{2} \\
&\left.\mp 24 A_{0} n^{3}+5 i n^{6}\right) .
\end{aligned}
$$

We have four nonlinear ODEs

$$
\begin{aligned}
a_{0} y_{z z z} & -30 a_{0} y^{2} \pm 4 a_{0} n y_{z z}+a_{0} n^{2} y_{z} \pm a_{0} n^{3} y \\
& +60 a_{0} A_{0} y+C_{1}=0, \\
a_{0} y_{z z z} & -30 a_{0} y^{2} \pm 4 i a_{0} n y_{z z}-a_{0} n^{2} y_{z} \mp i a_{0} n^{3} y \\
& +60 a_{0} A_{0} y+C_{1}=0,
\end{aligned}
$$

with exact solutions

$$
\begin{aligned}
& y(z)=A_{0} \pm n R+R_{z}, \\
& y(z)=A_{0} \pm i n R+R_{z} .
\end{aligned}
$$

The periodic solution (5.11) of 5.13 at $a_{0}=1, n=3$, $g_{2}=0.7485$ and $g_{3}=-0.2954$ is given in Figure 3 .

Equation (5.9) can be found from the nonlinear evolution equation

$$
u_{t}+\alpha u u_{x}+\beta u_{x x}+\gamma u_{x x x}+\delta u_{x x x x}=0 .
$$

This is the Kuramoto-Sivashinsky equation [46-48]. Equation (5.13) is used for the description of turbulence processes [ $48-50]$. Solutions of this equation are found in $[10,12,14,15]$.

From (5.9) and (5.10) one can see there is the special solution of equation (5.13) at $\beta=0$ and $\gamma=0$. This equation reminds the Burgers equation and takes the form

$$
u_{t}+\alpha u u_{x}+\delta u_{x x x x}=0 .
$$

Exact solutions of (5.14) are found by the formula

$$
y(z)=A_{0}+R_{z}
$$

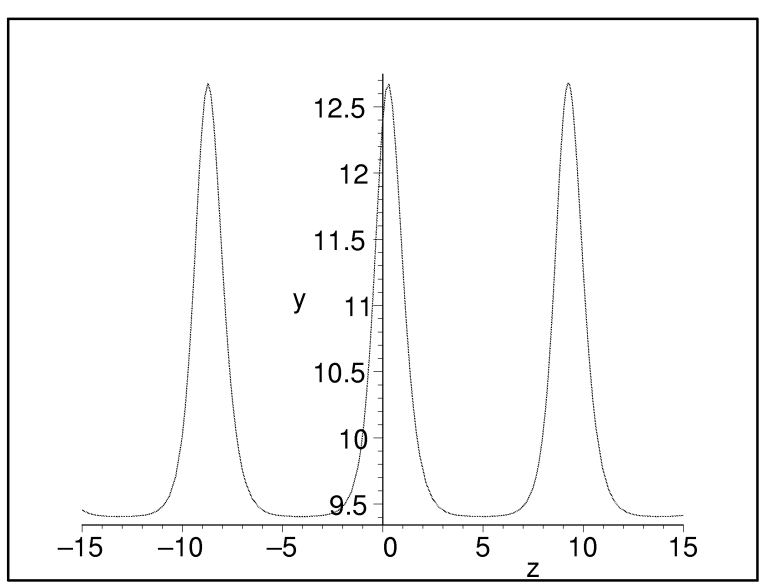

Fig. 3. Periodic solution (5.11) of Eq. (5.9) at $a_{0}=1, n=3$, $g_{2}=0.7485$ and $g_{3}=-0.2954$.

if we look for solution of (5.14) in the form of travelling waves. This equation takes the form

$$
\delta y_{z z z z}+\frac{\alpha}{2} y^{2}-C_{0} y+\frac{C_{0}^{2}+2160 \delta^{2} g_{3}}{2 \alpha}=0 .
$$

There is a rational solution of (5.16) in the form

$$
y(z)=\frac{C_{0}}{\alpha}+\frac{120 \delta}{\alpha\left(z-z_{0}\right)^{3}} .
$$

In this case (5.16) can be written in the simple form

$$
\delta y_{z z z z}+\frac{\alpha}{2} y^{2}-C_{0} y+\frac{C_{0}^{2}}{2 \alpha}=0 .
$$

We found nonlinear ODEs that correspond to the Kuramoto-Sivashinsky equation. These equations are very popular for the description of turbulence processes.

Fourth order ODEs. Let us find the nonlinear ODEs of fourth order with exact solutions (5.1). We can use the following general form of fourth order

$$
\begin{aligned}
a_{0} y_{z z z z} & +a_{1} y y_{z}+b_{0} y_{z z z}+b_{1} y^{2}+d_{0} y_{z z} \\
& +h_{0} y_{z}-g_{2} C_{0} y+C_{1}=0
\end{aligned}
$$

Assuming $A_{2}=-60$ and substituting (5.1) at $g_{2}=6 A_{2}^{4}$ into (5.19), we obtain at $A_{1}=0$ relations for the parameters:

$$
\begin{aligned}
& a_{1}=a_{0}, \\
& d_{0}=-\frac{1}{6} b_{1} A_{2}+\frac{1}{60} b_{0} A_{2}+\frac{1}{720} a_{0} A_{2}^{2},
\end{aligned}
$$




$$
\begin{aligned}
h_{0} & =4320 a_{0} A_{2}{ }^{3}-360 A_{2}{ }^{3} C_{0}, \\
b_{1} & =\frac{1}{2} b_{0}+\frac{1}{30} a_{0} A_{2} \\
b_{0} & =\frac{1}{12} A_{2}\left(186623999 a_{0}-15552000 C_{0}\right), \\
C_{0} & =12 a_{0} \\
C_{1} & =-18 a_{0} A_{2} g_{3}+\frac{1}{240} A_{2}^{7} a_{0} .
\end{aligned}
$$

Taking these values for the parameters into account we have a nonlinear ODE in the form

$$
\begin{aligned}
a_{0} y_{z z z z} & -\frac{1}{12} A_{2} a_{0} y_{z z z}+\frac{1}{720} a_{0} A_{2}^{2} y_{z z} \\
+ & a_{0} y y_{z}-\frac{1}{120} a_{0} A_{2} y^{2}-72 A_{2}^{4} a_{0} y \\
& -18 a_{0} A_{2} g_{3}+\frac{1}{240} A_{2}^{7} a_{0}=0 .
\end{aligned}
$$

Equation (5.27) can be found from the nonlinear evolution equation

$$
\begin{gathered}
u_{t}+\alpha\left(u u_{x}\right)_{x}+\beta u u_{x} \\
+\gamma u_{x x}+\delta u_{x x x}+\varepsilon u_{x x x x}+u_{x x x x x}=0,
\end{gathered}
$$

if we look for exact solutions in the form of travelling waves (1.2).

Solutions of this equation are expressed by

$$
y(z)=A_{2} R-60 R_{z}
$$

\section{Nonlinear ODEs with Exact Solutions of the Fourth Order Singularity}

Let us find nonlinear ordinary differential equations which have exact solutions of the fourth order singularity. These solutions can be presented by the formula

$$
y(z)=A_{0}+\frac{A_{1} R_{z}}{R}+A_{2} R+A_{3} R_{z}+A_{4} R^{2},
$$

where $R(z)$ satisfies the Weierstrass elliptic equation again.

We can not suggest nonlinear ODEs of the second and third order of the polynomial form with a solution (6.1). However we can consider the nonlinear fourth order ODE that takes the form

$$
a_{0} y_{z z z z}+a_{1} y^{2}+d_{0} y_{z z}+h_{0} y_{z}-g_{2} C_{0} y+C_{1}=0 .
$$

For calculations it is convenient to use $A_{1}=0, A_{3}=0$ and $A_{4}=-180$ :

$$
y(z)=A_{0}+A_{2} R-180 R^{2} .
$$

Substituting (6.1) into (6.2) we have

$$
\begin{aligned}
h_{0}= & 0, \quad a_{1}=\frac{14}{3} a_{0}, \quad d_{0}=-\frac{13}{30} a_{0} A_{2}, \\
A_{0}= & \frac{3}{28} \frac{g_{2} C_{0}}{a_{0}}+\frac{31}{25200} A_{2}^{2}+18 g_{2}, \\
g_{3}= & -\frac{1}{58320000} A_{2}\left(31 A_{2}^{2}-226800 g_{2}\right), \\
C_{1}= & -\frac{127}{300} a_{0} A_{2}^{2} g_{2}-\frac{961}{136080000} a_{0} A_{2}^{4} \\
& +\frac{3}{56} \frac{g_{2}^{2} C_{0}^{2}}{a_{0}}-1242 a_{0} g_{2}^{2}+168 a_{0} A_{2} g_{3} .
\end{aligned}
$$

In this case we get

$$
\begin{aligned}
a_{0} y_{z z z z} & +\frac{14}{3} a_{0} y^{2}-\frac{13}{30} a_{0} A_{2} y_{z z}-g_{2} C_{0} y \\
& -\frac{127}{300} a_{0} A_{2}^{2} g_{2}-\frac{961}{136080000} a_{0} A_{2}^{4} \\
& +\frac{3}{56} \frac{g_{2}^{2} C_{0}^{2}}{a_{0}}-1242 a_{0} g_{2}^{2}+168 a_{0} A_{2} g_{3}=0
\end{aligned}
$$

with the exact solution

$$
y(z)=A_{0}+A_{2} R-180 R^{2} .
$$

Equation (6.8) corresponds to the nonlinear evolution equation $[40,41,45]$

$$
u_{t}+\alpha u u_{x}+\beta u_{x x x}=\delta u_{x x x x x} .
$$

Exact solutions of (6.8) were found before [10,13] and rediscovered in a number of papers later.

At $A_{2}=0$ from (6.8) we have a nonlinear ODE of the form

$$
\begin{gathered}
a_{0} y_{z z z z}+\frac{14}{3} a_{0} y^{2}-g_{2} C_{0} y-1242 a_{0} g_{2}^{2} \\
+\frac{3}{56} \frac{g_{2}^{2} C_{0}^{2}}{a_{0}}=0 .
\end{gathered}
$$

This equation can be found from the nonlinear evolution equation $[41,45]$

$$
u_{t}+\alpha u u_{x}+\delta u_{x x x x x}=0
$$


if we look for exact solutions of this nonlinear evolution equation in the form of the travelling wave. Special solution of (6.11) and (6.12) are found by (6.9) at $A_{2}=0$.

\section{Nonlinear ODE with Exact Solution of the Fifth Degree Singularity}

We have not got any possibility to suggest a nonlinear differential equation of the polynomial class of the second, third and fourth order with an exact solution of the fifth degree singularity. We can look for such type of nonlinear fifth order ODE.

Assume we want to have a nonlinear ODE with exact solution of the fifth degree singularity

$$
\begin{aligned}
y(z)= & A_{0}+\frac{A_{1} R_{z}}{R}+A_{2} R+A_{3} R_{z} \\
& +A_{4} R^{2}+A_{5} R R_{z} .
\end{aligned}
$$

The simplest case of nonlinearity for this solution takes the form $y^{2}$, and we can see that (7.1) gives a singularity of the tenth degree. The general equation takes the form

$$
\begin{aligned}
\varepsilon y_{z z z z z} & +\gamma y_{z z z z}+\delta y_{z z z}+\chi y_{z z}+\beta y_{z}+\alpha y^{2} \\
& -C_{0} y+C_{1}=0 .
\end{aligned}
$$

This equation can be found from the nonlinear evolution equation $[18,51]$

$$
\begin{aligned}
& u_{t}+2 \alpha u u_{x}+\beta u_{x x}+\chi u_{x x x}+\delta u_{x x x x} \\
& +\gamma u_{x x x x x}+\varepsilon u_{x x x x x x}=0,
\end{aligned}
$$

if we search exact solutions in the form of travelling waves (1.2).

Consider the simplest case of (7.3) taking $\gamma=0, \chi=$ 0 and $\alpha=\frac{1}{2}$ into account. In this case, from (7.2) we get

$$
\varepsilon y_{z z z z z}+\delta y_{z z z}+\beta y_{z}+\frac{1}{2} y^{2}-C_{0} y+C_{1}=0 .
$$

Let us find exact solutions of the nonlinear ODE (7.4). At $A_{1}=0, A_{2}=0, A_{4}=0$ we have the following relations for the constants:

$$
\begin{aligned}
& A_{5}=-15120 \varepsilon, \quad A_{3}=-\frac{1260}{11} \delta, \\
& \beta=\frac{10}{121} \frac{\delta^{2}}{\varepsilon},
\end{aligned}
$$

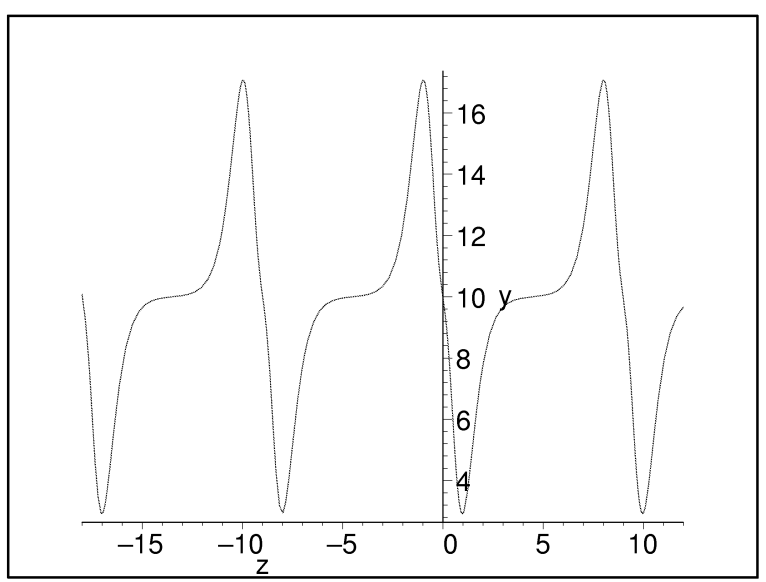

Fig. 4. Periodic solution (7.10) of Eq. (7.9) at $C_{0}=10.0$, $\delta=0.02, \varepsilon=0.001, g_{2}=0.7485$ and $g_{3}=-0.2954$.

$$
\begin{gathered}
g_{3}=-\frac{7}{660} \frac{\delta g_{2}}{\varepsilon}-\frac{1}{574992} \frac{\delta^{3}}{\varepsilon^{3}}, \\
g_{2}=\frac{1}{1452} \frac{\delta^{2}}{\varepsilon^{2}}+\frac{1}{5082} \frac{\delta^{2} \sqrt{21}}{\varepsilon^{2}}, \\
C_{1}=-\frac{10854}{161051} \frac{\delta^{5}}{\varepsilon^{3}}-\frac{2484}{161051} \frac{\delta^{5} \sqrt{21}}{\varepsilon^{3}}+\frac{1}{2} C_{0}{ }^{2} .
\end{gathered}
$$

Substituting (7.6) and (7.8) into (7.2) we obtain a nonlinear ODE in the form

$$
\begin{gathered}
\varepsilon y_{z z z z z}+\delta y_{z z z}+\frac{10}{121} \frac{\delta^{2} y_{z}}{\varepsilon}+\frac{1}{2} y^{2}-C_{0} y+\frac{1}{2} C_{0}^{2} \\
-\frac{10854}{161051} \frac{\delta^{5}}{\varepsilon^{3}}-\frac{2484}{161051} \frac{\delta^{5} \sqrt{21}}{\varepsilon^{3}}=0
\end{gathered}
$$

with the exact solution

$$
y(z)=C_{0}-\frac{1260}{11} R_{z}(\delta+132 \varepsilon R)
$$

where $R(z)$ satisfies the Weierstrass elliptic equation

$$
\begin{aligned}
R_{z}^{2} & -4 R^{3}+\left(\frac{1}{1452} \frac{\delta^{2}}{\varepsilon^{2}}+\frac{1}{5082} \frac{\delta^{2} \sqrt{21}}{\varepsilon^{2}}\right) R \\
& -\frac{7}{660} \frac{\delta g_{2}}{\varepsilon}-\frac{1}{574992} \frac{\delta^{3}}{\varepsilon^{3}}=0 .
\end{aligned}
$$

The periodic solution (7.10) of equation (7.9) at $C_{0}=10.0, \delta=0.02, \varepsilon=0.001, g_{2}=0.7485$ and $g_{3}=$ -0.2954 is given in Figure 4.

Equation (7.9) is found from the nonlinear evolution equation

$$
u_{t}+u u_{x}+\beta u_{x x}+\delta u_{x x x x}+\varepsilon u_{x x x x x x}=0 .
$$


In the case $\delta=0$ we obtain $\beta=0$ and have the rational solution of (7.12). This solution takes the form

$$
y(z)=C_{0}+\frac{30240 \varepsilon}{\left(z+z_{0}\right)^{5}} .
$$

Equation (7.9) in this case becomes

$$
\varepsilon y_{z z z z z}+\frac{1}{2} y^{2}-C_{0} y+\frac{1}{2} C_{0}^{2}=0 .
$$

In the last years (7.12) was used for the description of model chaos [52-54]. We hope that the exact solution (7.10) will be useful in the study of the turbulence processes where (7.12) is applied.

The rational solutions (5.17) and (7.13) of (5.18) and (7.14) suggest how one can find rational solutions of the generalized differential equations

$$
u_{t}+\alpha u u_{x}+\varepsilon u_{n+1, x}=0, \quad u_{n+1, x}=\frac{\partial^{(n+1)} u}{\partial x^{(n+1)}} .
$$

From (7.15) the nonlinear ODE becomes

$$
\varepsilon y_{n, z}+\frac{\alpha}{2} y^{2}-C_{0} y+\frac{C_{0}^{2}}{2 \alpha}=0, \quad y_{n, x}=\frac{d^{n} y}{d x^{n}} .
$$

Rational solutions of (7.16) take the form

$$
y(z)=\frac{C_{0}}{\alpha}+\frac{2(-1)^{(n-1)}(2 n-1) ! \varepsilon}{\alpha(n-1) !\left(z+z_{0}\right)^{n}} .
$$

[1] M. Musette and R. Conte, Physica D 181, 70 (2003).

[2] S. K. Liu, Z. T. Fu, and S. D. Liu et al., Phys. Lett. A. 309, 234 (2003).

[3] Z. Y. Yan, Chaos Solitons Fractals 15, 575 (2003).

[4] Z. Y. Yan, Chaos Solitons Fractals 15, 891 (2003).

[5] S. A. Elwakil, S. K. Ellabany, and M. A. Zahran et al., Phys. Lett. A 299, 179 (2002).

[6] E. G. Fan, Nuovo Cim. B 116, 1385 (2001).

[7] Z. T. Fu, S. K. Liu, and S. D. Liu et al., Phys. Let. A 290, $72(2001)$.

[8] E. G. Fan and L. Chao, Phys. Lett. A 285, 373 (2001).

[9] N. A. Kudryashov and M. B. Soukharev, J. Appl. Math. Mech. 65, 855 (2001).

[10] N.A. Kudryashov, Journal of Applied Mathematics and Mechanics, 52, 361 (1988).

[11] R. Conte and M. Musette, J. Phys. A 22, 169 (1989).

[12] N. A. Kudryashov, Phys Lett. A 147, 287 (1990).

[13] N. A. Kudryashov, Phys Lett. A 155, 269 (1991).

[14] N. G. Berloff and L. N. Howard, Stud. Appl. Math. 100, 195 (1998).
Here $z_{0}$ is an arbitrary constant. The exact solution (7.17) can be useful in numerical simulations of nonlinear problems where (7.15) and (7.16) are used.

\section{Conclusion}

We have found a polynomial class of nonlinear differential equations of the second, third and fourth order which have exact solutions expressed by the Weierstrass function. These solutions have different singularities and are expressed via general solutions of the Weierstrass elliptic equation.

We also list a number of nonlinear ODEs with exact solutions. These equations are found from the widely used nonlinear evolution equations taking the travelling wave into account.

\section{Acknowledgements}

This work was supported by the International Science and Technology Center under Project No 1379-2. The material is partially based upon work supported by the Russian Foundation for Basic Research under Grant No 01-01-00693.

[15] N. A. Kudryashov, Analytical theory of nonlinear differential equations, Moscow-Igevsk, IKI (2004), 360 p.

[16] S. R. Choudhury, Phys. Lett. A 159, 311 (1997).

[17] R. Conte and M. Musetem, J. Phys. A. Math. Gen. 25, 5609 (1992).

[18] N. A. Kudryashov, Mathematical simulation 1, 55 (1989), in Russian.

[19] N. A. Kudryashov and E.D. Zargaryan, J. Phys. A. Math. and Gen 29, 8067 (1996).

[20] E. G. Fan, Phys Lett. A 227, 212 (2000).

[21] S. A. Elwakil, S. K. Ellabany, and M. A. Zahran et al., Z. Naturforsch. 58, 39 (2003).

[22] N.A. Kudryashov, Nonlinear differential equations with exact solutions, arXiv:nlin.SI/0311058 v.1; 27 Nov (2003).

[23] N. A. Kudryashov, Mathematical simulation, 1, 151 (1989), in Russian.

[24] Z. Y. Yan, Z. Naturforsch. 59a, 29 (2004).

[25] Z. Y. Yan, Chaos, Solitons and Fractals 21, 1013 (2004). 
[26] Z. Y. Yan, J. Phys. A 36, 1961 (2003).

[27] Z. Y. Yan, Comput. Phys. Commun. 153, 1 (2003).

[28] P. Painleve, Acta Math. 25, 54 (1902).

[29] H. Aspe and M. C. Depassier, Phys. Rev. A 41, 3125 (1990).

[30] A. Garazo and M. G. Velarde, Phys. Fluids A 3, 2295 (1991).

[31] D. E. Bar and A. A. Nepomnyaschy, Physica D 86, 586 (1995).

[32] A. A. Nepomnyaschy, Trans. Perm. State Univ. 362, 114 (1976), in Russian.

[33] S. Y. Lou, G. X. Huang and H. Y. Ruan, J. Phys. A Math. Gen. 24, L587 (1991).

[34] A. V. Porubov, J. Phys. A. Math. Gen. 26, L797 (1993).

[35] P. J. Caudrey, R. K. Dodd, and J. D. Gibbon, Proc. Roy Soc. London A 351, 407 (1976).

[36] J. Weiss, J. Math. Phys. 25, 13 (1984).

[37] B. Kuperschmidt and G. Wilson, Invent. Math. 62, 403 (1981).

[38] N. A. Kudryashov, J. Phys. A. Math. Gen. 32, 999 (1999).

[39] N. A. Kudryashov, J. Nonl. Math. Phys. 8, 172 (2001).

[40] V. I. Olver, Hamiltonian and non-Hamiltonian models for water waves, in Lecture notes in Physics, No., Springer-Verlag, New York 1984, p. 273.
[41] A. I. Zemlyanukhin and L. I. Mogilevich, Acoust. Phys. 47, 303 (2001).

[42] T. Kawahara and S. Toh, Phys. Fluids 31, 2103 (1988).

[43] B.F. Feng and T. Kawahara, J. Bifurcat. Chaos, 12, 2393 (2002).

[44] Y. Yamamoto and E. I. Takizawa, J. Phys. Soc. Japan 50, 1055 (1981)

[45] V. I. Karpman, Phys. Lett. A 244, 8035 (1998).

[46] Y. Kuramoto and T. Tsuzuki, Prog. Theor. Phys. 55, 356 (1076).

[47] G. I. Sivashinsky, 4, 227 (1982).

[48] P. S. Landa, Nonlinear Oscillations and Waves in Dynamical Systems, Kluwer Academic Publishers, Dordrecht 1996, p. 538.

[49] C.M. Alfaro, R.D. Benguria, and M.C. Depassier, Physica D 61, 1 (1992).

[50] T. Kawahara, Phys. Rev. Lett. 51, 381 (1983).

[51] L. A. Beresnev and, V. N. Nikolaevskiy, Physyca D 66, 1 (1993).

[52] M. I. Tribelsky and K. Tsuboi, Phys. Rev. Lett. 76, 1631 (1996).

[53] H.-W. Xi, R. Toral, J. D. Gunton, and M. I. Tribelsky, Phys. Rev E. 62, 17 (2000).

[54] R. Toral, G. Xiong, J. D. Gunton, and H.-W. Xi, J. Phys. A. Math. Gen. 36, 1323 (2003). 